\title{
Descripción de hábitos compra y endeudamiento en una muestra de consumidores del área metropolitana de Pereira*
}

\author{
Felipe Parrado ${ }^{1}$ \\ Universidad Católica de Pereira (Colombia) \\ Magda Mendivelso² \\ Universidad Cooperativa de Colombia, sede Villavicencio (Colombia)
}

Recibido: mayo 5 de 2015 - Revisado: junio 16 de 2015 - Aceptado: agosto 30 de 2015

Referencia formato APA: Parrado, F., \& Mendivelso, M. (2015). Descripción de hábitos compra y endeudamiento de una muestra de consumidores del área metropolitana de Pereira. Revista Científica Guillermo de Ockham, 13(2), 107-115.

\section{Resumen}

La repetición del consumo es una condición importante no solo en la dinámica de la economía, sino en el bienestar que genera al satisfacer las necesidades las personas. El presente estudio pretende describir los hábitos de consumo de una muestra de habitantes de Pereira y su área metropolitana. Para esto se siguió un diseño no experimental transversal, con muestreo intencional y se tomaron como criterios de inclusión a personas mayores de edad y económicamente activas. Los instrumentos empleados fueron la escala de hábitos y conductas de consumo, un cuestionario sociodemográfico y tres preguntas que indagan sobre el ingreso mensual, el número de tarjetas de crédito que tiene el participante y la percepción de la situación financiera. Los resultados indican diferencias estadísticamente significativas entre los grupos en las categorías de hábitos de planificación de consumo ( $p>, 001)$, hábitos de compra ( $p>, 001$ ) y hábitos de uso de crédito ( $p>, 001)$. Se discuten los resultados a la luz de su importancia para el desarrollo económico y el bienestar. Se plantean las limitaciones del estudio y la posibilidad relacionar este constructo con el bienestar psicológico.

Palabras clave: Consumidor, hábito de consumo, planificación, compra, crédito, bienestar

\section{Description of shopping and indebtedness habits of a sample of consumers of the metropolitan area of Pereira}

\section{Abstract}

Repetition of consumption is an important condition not only in the dynamics of the economy but also for the welfare generated to meet the needs of people. This research aims at describing the habits from a sample of people in Pereira

\footnotetext{
* El presente artículo es resultado del proyecto de investigación Autorregulación de la conducta. Compra y consumo cuyo objetivo era identificar problemas de regulación de comportamiento en las relaciones contextuales del consumidor en el Eje Cafetero. El proyecto fue financiado por la Universidad Católica de Pereira en el periodo 2013-1014.

1. Psicólogo, especialista en Psicología del Consumidor. Magíster en Cerebro y Conducta de la Universidad de Sevilla. Dirigir correspondencia al Laboratorio de Psicología Experimental, bloque Kabai, 2o. piso, Universidad Católica de Pereira. Av. de las Américas . 49-95 C. P. 660005. Pereira, Risaralda, Colombia. E-mail: felipe.parrado@ucp.edu.co

2. Psicóloga. Magíster en Psicodiagnóstico y Evaluación Psicológica de la Universidad de Buenos Aires. E-mail: magda.mendivelsod@campusucc.edu.co
} 
and its metropolitan area. For this, a transversal non-experimental design was followed, with purposive sampling, and using inclusion criteria which was being an adult and being economically active. The instruments used were the scale of consumption habits and behaviors, a socio demographic questionnaire and 3 questions which inquired about their monthly income, the number of credit cards owned by the participant and a perception of their financial situation. The results indicate statistically significant differences between groups in the categories of consumer habits planning ( $p>, 001)$, shopping habits $(\mathrm{p}>, 001)$ and credit usage habits $(\mathrm{p}>, 001)$. The results are discussed according to the importance of these for welfare and economic development. The limitations are pointed out and the possibility to relate this construct to psychological well-being.

Keywords: Consumer, consumer habits, planning, purchase, credit, well-being

\section{Descrição de compra e hábitos de empréstimos obtidos em uma amostra de consumidores na região metropolitan de Pereira}

\section{Resumo}

A repetição do consumo é uma condição importante não somente para a dinâmica da economia, mas também para em relação com bem-estar gerado ao atender às necessidades das pessoas. Este estudo visa descrever os hábitos de consumo de uma amostra de habitantes na cidade de Pereira e sua regiáo metropolitana. Para isso, foi implementado um delineamento transversal não experimental, com uma amostragem não-intencional que teve como critérios de inclusão pessoas maiores de idade e económicamente ativas. Os instrumentos utilizados foram uma escala de hábitos e condutas de consumo, um questionário sócio demográfico e 3 perguntas que indagaram a renda mensal, o número de cartôes de crédito possuidos pelo participante, assim como a prercepção da situação financeira. Os resultados indicam diferenças estatísticamente significativas entre os grupos nas categorias de hábitos de planejamento de consumo ( $p>, 001)$, hábitos de compra ( $p>, 001)$ e hábitos de uso de crédito (p>,001). Os resultados são discutidos à luz da sua importância a respeito do desenvolvimento económico e do bem-estar. As limitaçóes do estudo sao apresentadas e a possibilidade de relacionar este construto com o bem-estar psicológico.

Palavras-chave: Consumidor, hábito de consumo, planejamento, compra, crédito, bem-estar

\section{Introducción}

El comportamiento de consumo se define a partir de las acciones dirigidas a la compra, uso y disposición de bienes y servicios para satisfacer sus necesidades (Schiffman \& Kanuk, 2005). La investigación de este campo se ha centrado en los aspectos psicológicos y sociales que preceden y siguen a estos procesos (Henao \& Cordoba, 2007; Velandia \& López, 2008). El proceso de consumo se ha abordado en un orden temporal definido, fuera de que es un comportamiento que tiende a repetirse como parte natural del sistema económico en el cual vivimos. Así, se pueden identificar una cadena de conductas ejecutadas en varias etapas: la selección, la compra, el uso y el desecho (Solomon, 2008).

Para entender la repetición de conductas encadenadas y automáticas han surgido múltiples conceptos en la psicología, uno de los cuales es el concepto de hábito. Los hábitos se adquieren gradualmente como una disposición a repetir una conducta de respuesta frente a un contexto recurrente y no necesariamente están regulados por una actividad cognitiva de alto orden. Los hábitos son un aspecto fundamental en la psicología del consumidor puesto que el $45 \%$ de las conductas cotidianas son repetidas diariamente y con frecuencia ocurren en el mismo contexto (Wood \& Neal, 2009).

Desde el punto de vista de la psicología de la salud, la definición del hábito recalca el automatismo de la acción más que la repetición, puesto que cabe la posibilidad de establecer hábitos en ausencia de un entrenamiento exhaustivo. Así, el hábito se compone de ejecución dependiente de una clave -cualidad central-y de las repeticiones dependientes del contexto -causa- (Gardner, 2012; Sniehotta \& Presseau, 2012). La automaticidad, una vez formada, no necesita una ejecución frecuente, así podría tener mayor poder explicativo dado que muchos hábitos a

$\varangle$ Universidad de San Buenaventura, Cali - Colombia 
pesar de ser repetitivos tienen ciclos de reiteración bastante extendidos, lo cual a ojos del observador serían apreciados como infrecuentes. Los hábitos en diversos ámbitos, se consideran como tipos específicos de automatismo caracterizado por el marcaje contextual rígido de la conducta que no depende de las metas y los objetivos de las personas (Gardner, 2014; Wood \& Neal, 2009).

La creación y modificación de hábitos es importante para la salud de las personas, pero también para el desarrollo de los mercados (Lally \& Gardner, 2011; Verplanken \& Wood, 2006). El automatismo y la repetición de acciones en un contexto pueden tener resultados nocivos (como en el caso de las adicciones, al tiempo que explican los patrones de selección, compra, uso y desecho de productos, bienes o servicios.

Para formar un hábito se requiere que el comportamiento sea ejecutado repetidamente en un contexto consistente. La formación de hábitos sigue ciertos pasos: primero, la necesidad de hacer una acción, luego una decisión que se convierte en acción, posteriormente, la conducta tiene que ser repetida y requiere de motivación continua hasta el desarrollo del automatismo. Por otro lado, para romper los hábitos los autores señalan que se requerirá interrumpir la asociación entre señal-respuesta, descontinuar la exposición a las claves o señales del hábito o presentar alternativas de respuesta a dichas claves -estímulos novedosos- (Lally \& Gardner, 2011; Gardner, 2014).

Además del abordaje con base en los estudios de mercadeo y economía comportamental, el consumo y el endeudamiento habituales se han trabajado en áreas como la antropología, la economía y la sociología, dado que es innegable la influencia de la cultura, los fenómenos económicos (inflación) y las regulaciones del comportamiento derivados de la vida en grupo. Estas disciplinas se han interesado en el conocimiento de los objetos, los actores, las relaciones, las prácticas y los lugares de consumo, por lo cual aportan una perspectiva diferente para comprender de la repetición de las acciones humanas.

La antropología, por su parte, se interesa en investigar la cultura del consumo en diversas manifestaciones. A nivel macro, su énfasis se pone en los aspectos que influyen en la identidad del consumidor, como la economía y la cultura en la que se encuentra, y a nivel micro se centra en conocer los sistemas de producción en culturas específicas (Bruneau Valenzuela, Marques de Mello \& David Vieira, 2009).

De otro lado, la sociología se enfoca en el contexto social del consumidor y la influencia de diferentes variables sobre el consumo, entre las que se encuentran los grupos de referencia-familiares, amigos, compañeros de trabajo-, la clase social, la cultura y los valores que esta considera importantes, y las subculturas a las que pertenece, como la nacionalidad, la religión y la edad (Schiffman \& Kanuk, 2005).

En cuanto a la teoría económica, esta se ha acercado al consumo a partir de modelos que intentan explicar su comportamiento en relación con la producción y la deuda. Según Briceño, Jiménez y Muñoz (2015), tres teorías económicas principales explican el consumo: la teoría del ingreso relativo, la teoría del ciclo de vida y la teoría del ingreso permanente. En todo caso se ha descrito que el consumo tiene un comportamiento diferente a corto y largo plazo.

La teoría del ingreso relativo señala que el consumo está determinado por la posición relativa del consumidor en la distribución del ingreso, por lo cual el ahorro, la deuda y el gasto están determinados por el ingreso.

La teoría del ciclo de vida aduce que el consumo depende del momento vital del consumidor y de su ingreso en ese momento. Los comportamientos de gasto, ahorro y deuda están determinados por la experiencia inmediata con el dinero.

La teoría del ingreso permanente postula que el consumidor ajusta su gasto a las expectativas de ingreso a largo plazo, por lo que el individuo considera normal ciertos niveles de consumo, gasto y ahorro de acuerdo a su grupo social.

Aunque el papel del consumidor en estas teorías parece ser secundario, el comportamiento es fundamental para comprender los efectos macroeconómicos, tal como la relación entre ingreso y consumo. El dato reportado por Briceño (2014) indica que con respecto a otros países latinoamericanos, Colombia no presenta una relación equilibrada entre consumo e ingreso; por el contrario, hemos desarrollado patrones de consumo más estables a pesar de las variaciones de ingreso de las últimas dos décadas.

Por su parte, Rodríguez (2006) plantea un modelo piscoeconómico que puede ser útil para comprender esta diferencia en el caso colombiano, en él se consideran las actitudes hacia la deuda, el ingreso, el bienestar subjetivo y los hábitos como determinantes de la conducta de consumo y deuda. Integrar constructos psicológicos a las variables económicas lleva a comprender mejor los hábitos y su importancia para la vida diaria (Rayo \& Becker, 2007). 
Además de los elementos contextuales y económicos, los consumidores forman hábitos basados en su experiencia individual. A partir de su estudio del consumidor, Verplanken y Wood (2006) proponen dos tipos de intervención sobre los hábitos: la adición hacia abajo, que consiste en proveer información sobre la acción en momentos donde el hábito es susceptible de cambiar, como cuando las personas cambian naturalmente de ambientes y de ejecución (mudarse de casa, cambio de trabajo); y las intervenciones hacia arriba, que ocurrirían antes de la ejecución del hábito y prevendrán la aparición de las claves ambientales relacionadas con el hábito para establecer unas nuevas.

Así, quienes diseñan políticas de intervención sobre el consumidor deberían tener en cuenta no solo el cambio de los hábitos en sí, sino también la adquisición y el mantenimiento de nuevas conductas mediante la formación de nuevos hábitos que reemplacen o compitan con los antiguos (Verplanken \& Wood, 2006).

\section{Método}

\section{Participantes}

La muestra de participantes estuvo compuesta por 471 personas seleccionadas mediante muestreo no probabilístico intencional, durante el primer semestre de 2014. Cada uno de los participantes fue contactado al azar, de forma personal por estudiantes del programa de Mercadeo de la Universidad Católica de Pereira, quienes durante un día de la semana debían ubicarse en un lugar concurrido de la ciudad y entrevistar a veinte personas que cumplieran con los criterios de la muestra.

Los criterios de inclusión en el estudio fueron: sujetos mayores de edad y económicamente activos. La edad media de los participantes fue de 35,98 años ( $\mathrm{DE}=15,9)$, de los cuales el $52,9 \%$ fueron mujeres y $47,1 \%$ hombres.

Respecto a la ciudad de residencia el $81 \%$ de la muestra fue de Pereira, $15 \%$ de Dosquebradas, 2,3 \% de Santa Rosa de Cabal, y 1,5 \% de Cartago. El nivel socioeconómico de la muestra fue: 7,4 \% para el estrato $1 ; 26,5 \%$ para el estrato $2 ; 36,5 \%$ para el estrato $3 ; 18,7 \%$ para el estrato $4 ; 8,9 \%$ para el estrato 5 y $1,9 \%$ para el estrato 6 (la moda es el estrato 3). Así mismo, el 80,7 \% de los participantes se encontraban empleados y el 19,3\% desempleados al momento de la aplicación de las encuestas. Además, el $61 \%$ de la muestra reportó tener personas a cargo y el 39 \% informó que no era responsable económicamente de nadie.

\section{Instrumentos}

Respecto de la escala de hábitos y conductas de consumo se usó la versión adaptada por Ortega y RodríguezVargas (2004) y usada posteriormente por Ortega, Vargas, Denegri y Gempp (2005) en Barranquilla, Colombia. Este instrumento tiene buenas evidencias de validez de contenido (juicio experto y prueba piloto) y de constructo.

\section{Procedimiento de aplicación}

Al hacer la entrevista y antes de contestar los cuestionarios, se le informó a cada participante sobre los objetivos del estudio y las personas que participaron firmaron un consentimiento informado. Las respuestas de los participantes a cada cuestionario se registraron en papel. Luego de finalizar se les agradeció su participación y se les indicó información de contacto para obtener retroalimentación sobre los resultados.

Posteriormente, los estudiantes transcribieron las respuestas y se compilaron en una base de datos digital gestionada en Google Docs. Los datos fueron descargados y procesados posteriormente con el paquete estadístico SPSS v.20. Se efectuaron análisis descriptivos para las variables sociodemográficas y se calculó la independencia de las opciones de respuesta respecto de las variables planificación de compra, compra y endeudamiento, para describir los perfiles de hábitos en la muestra de participantes.

\section{Procedimiento de medición}

El formato de respuesta era una escala Likert de tres opciones (sí, no, a veces), sobre los siguientes ítems que evalúan las conductas de compra referidos a la frecuencia con que se realizan: 1 . hace una lista de los productos y servicios que necesita comprar; 2 . selecciona los productos según su calidad; 3. para distribuir su dinero ordena los productos según su importancia antes de comprar; 4. selecciona los productos según su precio; 5 . compara precios entre distintas marcas; 6. compara precios en distintos locales de venta; 7. lee las etiquetas de todos los productos; 8. observa el peso neto de los productos; 9. se asegura de que los electrodomésticos tengan servicio técnico en la ciudad; 10. pregunta por el tiempo de garantía de los productos; 11. pregunta sus dudas al vendedor antes de comprar; 12. planifica sus compras con antelación; 13. si compra ropa, lee la etiqueta para saber las características y el cuidado que requiere; 14 . examina detalladamente todos los productos que compra; 15 . cree que gasta más de lo que debería; 16. compra en el comercio ambulante; 17. compra habitualmente a crédito; 18 . al solicitar crédito, pregunta por las tasas de interés y el incremento del precio 
final; 19. al comprar a crédito, compara las tasas de interés en distintos locales.

Adicionalmente, se emplearon tres ítems para evaluar las siguientes características:

\section{Ingreso}

Se evaluó con un ítem que pregunta por el ingreso personal mensual. El formato de respuesta es una escala Likert de ocho opciones de respuesta: menos de $\$ 400$ mil; entre $\$ 400$ y $\$ 700$ mil; entre $\$ 700$ y $\$ 1$ millón; entre $\$ 1$ y $\$ 1.5$ millones; entre $\$ 1.5$ y $\$ 2$ millones; entre $\$ 2$ y $\$ 3$ millones, y más de $\$ 3$ millones (Ortega et al., 2005).

\section{Posesión de tarjetas de crédito}

Se evaluó mediante un ítem que explora el número de tarjetas de crédito que tiene el evaluado. El formato de respuesta es abierto (Ortega et al., 2005).

\section{Percepción de la situación financiera}

Se evaluó con un ítem en el que los participantes describen su situación financiera. Tiene un formato de respuesta Likert de cuatro puntos: difícil, regular, aceptable y buena (Ortega et al., 2005).

\section{Cuestionario sociodemográfico}

Se evaluó mediante preguntas que exploran la edad, el sexo, la ciudad de residencia, el estrato socioeconómico, la situación laboral actual (empleado o desempleado), y si tenían o no personas a cargo.

Para garantizar la precisión de las mediciones, se exploró la confiabilidad por consistencia interna, un procedimiento estadístico que permite comprobar la homogeneidad de las puntuaciones y emplea el coeficiente alfa $(\alpha)$ de Cronbach (Virla, 2010). De esta manera, se exploró la confiabilidad por consistencia interna de la prueba total mediante el método de división por mitades y se obtuvo un $\alpha=0,66$, lo que indica la consistencia de las puntuaciones obtenidas. Con el fin de reflejar la cadena de habitual de consumo, esta se dividió en tres partes: la planificación, la compra y el crédito.

\section{Resultados}

Para comprobar si existen diferencias entre los grupos de respuesta se llevó a cabo la prueba estadística chi cuadrado $\left(\mathrm{X}^{2}\right)$ que permite corroborar si las diferencias en los datos se deben a diferencias estadísticamente significativas entre los grupos, o por el contrario, hay igualdad entre los grupos. Se empleó una prueba $\mathrm{X}^{2}(2, N=471), p<0.01$, y se encontró que para los ítems 1, 2, 3, 4, 5, 6, 7, 9, 10, 11, 13, 14, 15, 18 y 19, las frecuencias de respuesta (sí, no, a veces) son estadísticamente diferentes. Ninguna opción de respuesta $(0,0 \%)$ obtuvo frecuencias esperadas menores a 5 . Así mismo, la frecuencia de casilla esperada mínima fue de 157.

En la Tabla 1 se puede observar que $44,6 \%$ de las personas encuestadas hacen listas de los productos y servicios que van a comprar, además $55,4 \%$ ordenan dichos artículos para distribuir su dinero antes de comprar. Aquí cabe afirmar que la planificación de las compras es un hábito común dentro de la mayoría de muestra encuestada.

Sin embargo, los hábitos de compra no son tan homogéneos. Los resultados muestran que la mayoría de los encuestados alternan sus compras en el comercio informal $(34,2 \%)$. La mayoría examina detalladamente los productos que compra $(40,1 \%)$, aunque si se trata de ropa gran parte de ellos $(44,8 \%)$ no lee la etiqueta sobre sus características y cuidado, de la misma forma se descuida de leer las etiquetas $(37,2 \%$ y detalles como el peso de los productos $(31,4 \%)$, a pesar de esto, el 59,9\% afirma tener en cuenta la calidad de los productos a la hora de comprar. En lugar de la información de las etiquetas, parece ser que los consumidores recurren a información suministrada por el vendedor $(62,4 \%)$ y preguntan por el tiempo de garantía $(78,3 \%)$, el servicio técnico para electrodomésticos (49,3\%). El precio es un atributo muy valorado. El 58,2 \% de ellos lo toma como índice para la selección de un producto y así la mayor parte de la muestra compara precios en diferentes locales (44,8\%) $y$ diferentes marcas $(67,3 \%)$.

Respecto de la evaluación del ingreso de la población estudiada, se encontró que el 26,8 \% de los participantes tienen ingresos entre 400 y 700 mil pesos; el $21,4 \%$ entre 700 mil y 1 millón de pesos; el 15,3\% menos de 400 mil pesos; el $13,4 \%$ entre 1 y 1,5 millones de pesos; el $10 \%$ entre 1,5 y 2 millones de pesos; el $7 \%$ más de 3 millones de pesos, y el 6,2 entre 2 y 3 millones de pesos.

Para la posesión de tarjetas de crédito, se encontró que el 46,1 \% de la muestra no tiene tarjeta; el 25,7 \% tienen una tarjeta; el 13,6\% dos tarjetas; el 8,7 \% tres tarjetas; el $4,2 \%$ cuatro tarjetas y menos del $1 \%$ cinco, seis y siete tarjetas de crédito. Finalmente, en la percepción de la situación financiera, se encontró que el 35,5\% cree que es aceptable; el 30,6\% regular; el 23,6 \% buena, y el $10,4 \%$ difícil. 
Tabla 1

Hábitos de planificación, compra y endeudamiento de una muestra de habitantes de Pereira

\begin{tabular}{|c|c|c|c|c|c|}
\hline Hábitos de planificación de consumo & No $\%$ & Sí \% & A veces $\%$ & Chi cuadrado & Sig. \\
\hline Hace una lista de los productos y servicios que necesita comprar (Item 1). & 25,5 & 44,6 & 29,9 & 28,242 & 0,000 \\
\hline $\begin{array}{l}\text { Para distribuir su dinero, ordena los productos según su importancia antes } \\
\text { de comprar (Item 3). }\end{array}$ & 20 & 55,4 & 24,6 & 104,879 & 0,000 \\
\hline Planifica sus compras con antelación (Item 12). & 27,2 & 37,6 & 35,2 & 8,420 & 0,015 \\
\hline Hábitos de compra & No $\%$ & Sí \% & A veces $\%$ & Chi cuadrado & Sig. \\
\hline Selecciona los productos según su calidad (Item 2). & 10,2 & 59,9 & 29,9 & 176,828 & 0,000 \\
\hline Selecciona los productos según su precio (Item 4). & 14,2 & 58,2 & 27,6 & 143,427 & 0,000 \\
\hline Compara precios entre distintas marcas (Item 5). & 12,5 & 67,3 & 20,2 & 248,713 & 0,000 \\
\hline Compara precios en distintos locales de venta (Item 6). & 22,3 & 50,7 & 27 & 65,783 & 0,000 \\
\hline Lee las etiquetas de todos los productos (Item 7). & 37,2 & 24 & 38,9 & 18,701 & 0,000 \\
\hline Mira el peso neto de los productos (Item 8). & 31,4 & 36,3 & 32,3 & 1,924 & 0,382 \\
\hline $\begin{array}{l}\text { Se asegura de que los electrodomésticos tengan servicio técnico en la } \\
\text { ciudad (Item 9). }\end{array}$ & 26,5 & 49,3 & 24,2 & 54,127 & 0,000 \\
\hline Pregunta por el tiempo de garantía de los productos (Item 10). & 5,7 & 78,3 & 15,9 & 436,739 & 0,000 \\
\hline Pregunta sus dudas al vendedor antes de comprar (Item 11). & 10,2 & 62,4 & 27,4 & 200,217 & 0,000 \\
\hline $\begin{array}{l}\text { Si compra ropa, lee la etiqueta para saber las características y el cuidado } \\
\text { que requiere (Item 13). }\end{array}$ & 44,8 & 26,8 & 28,5 & 28,064 & 0,000 \\
\hline Examina detalladamente todos los productos que compra (Item 14). & 18,7 & 40,1 & 41,2 & 45,567 & 0,000 \\
\hline Compra en el comercio ambulante (Item 16). & 34,2 & 35,5 & 30,4 & 1,987 & 0,370 \\
\hline Hábitos de endeudamiento & No $\%$ & Sí \% & A veces $\%$ & Chi cuadrado & Sig. \\
\hline Cree que gasta más de lo que debería (Item 15). & 25,7 & 46,7 & 27,6 & 38,178 & 0,000 \\
\hline Compra habitualmente a crédito (Item 17). & 39,3 & 29,7 & 31 & 7,605 & 0,022 \\
\hline $\begin{array}{l}\text { Al solicitar crédito, pregunta por las tasas de interés y el incremento del } \\
\text { precio final (Item 18). }\end{array}$ & 31,2 & 54,4 & 14,4 & 113,516 & 0,000 \\
\hline $\begin{array}{l}\text { Al comprar a crédito, compara las tasas de interés en distintos locales } \\
\text { (Item 19). }\end{array}$ & 46,1 & 36,9 & 17 & 62,535 & 0,000 \\
\hline
\end{tabular}

Fuente: Elaboración propia.

En términos generales, al considerar la tendencia de las respuestas se evidencia que los participantes presentan una adecuada planeación de consumo, hábitos de compra y de endeudamiento. Sin embargo, resaltan los ítems 7, 13 y 19, en los cuales la tendencia no se cumplió, lo que indica que los participantes, en su mayoría, no leen las etiquetas de los productos que compran, no tienen en cuenta las recomendaciones para el cuidado de la ropa y a la hora de comprar a crédito no suelen comparar las tasas de interés en distintos lugares, aspectos relacionados con los hábitos de compra y endeudamiento respectivamente.

\section{Discusión}

Los resultados anteriores dan una visión acerca de la manera como se planifican las decisiones de consumo y de los hábitos de compra y deuda de los habitantes de Pereira, a pesar de que la muestra que se recogió corresponde a la población económicamente activa (66 \%) (Pereira, cómo vamos, 2015) en el periodo de estudio.

Müller (2009), hace un análisis etnográfico de las representaciones y prácticas económicas de las personas en Brasil y afirma que las personas en su toma de decisiones se guían por las normas y obligaciones frente a la familia, el sexo y la generación en que se encuentran. Así mismo, las acciones de las personas de bajos ingresos se basan principalmente en resolver de forma práctica sus problemas y para comprender los hábitos de compra y endeudamiento de una manera más integral, se deben tener en cuenta varios elementos entre los que se incluyen ingresos, patrones de consumo, clase social, historia familiar, experiencias de pobreza en la infancia, matrimonio, embarazo precoz e hijos, entre otros.

$112<$ Universidad de San Buenaventura, Cali - Colombia 
En este sentido, aunque los resultados corresponden a una muestra limitada, se puede notar que la mayoría de las personas encuestadas tiene un hábito establecido para planificar la compra (listar los productos y ordenar en importancia). Sin embargo muchos hacen compras no planificadas y consumen en el sector informal. En el área metropolitana de Pereira, a pesar de su historia y desarrollo económico, el consumo en lugares informales forma parte del panorama cotidiano de la ciudad (Galvis, 2012).

Comprar en un lugar informal no demerita que al momento de llevar a cabo los intercambios la mayoría de los consumidores encuestados tienen en cuenta la calidad, el precio y la marca, como los atributos relevantes para decidir la selección de un producto. Esta observación estaría en consonancia con las diferencias entre expectativas y realidades de los lugares de consumo en Pereira seńaladas por Rubiano (2010), quien sostiene que las personas perciben los centros comerciales como un símbolo de renovación urbana y modernidad. Pero los hábitos de consumo no se ajustan del todo a esa percepción. Cabe recordar que la definición del hábito no implica la búsqueda de objetivos, sino un reflejo de la automatización de la acción. En este caso, la compra en canales informales automatiza los intercambios cotidianos de las personas de la región.

En cuanto al uso del crédito y medios de pago, se resaltar que gran parte de los encuestados $(40 \%)$ no tiene tarjeta de crédito y sus ingresos se ubican en medio-bajos, lo cual explica por qué pocos conocen de las tasas de interés. Aun así, la mayoría repara en que gasta más de lo que debiera. Estos resultados apoyan las predicciones del modelo psicoeconómico del consumidor propuesto por Rodríguez (2006), que predice que los individuos de menores ingresos y un menor estado de endeudamiento tendrán una percepción subjetiva más pesimista de la situación financiera.

Aunque dicho modelo psicoeconómico pronostica que las conductas impulsivas se relacionan con la percepción financiera pesimista, los resultados de este estudio no apoyan esta predicción del modelo con respecto a los hábitos y conductas de consumo impulsivas, dado que la mayoría de la población encuestada reporta estrategias de planificación y regulación de la compra (comparar, preguntar, etc.). Aun así, estas acciones se muestran poco eficaces, pues la mayoría de las personas no tiene una buena percepción de su situación financiera y afirma gastar más de lo que debiera.

Esta situación, sumado al bajo nivel de ingresos, a la poca experiencia o conocimiento del crédito y a la promoción de la región como una zona de comercio y consumo, podría deteriorar el bienestar de los habitantes del área metropolitana de Pereira al hacer más notorias las claves de repetición y automatización del consumo, sin que se presente una mejoría del poder adquisitivo. Si se asume que el bienestar y la felicidad son el resultado de la toma de decisiones de las personas -como argumentan Ocampo (2014), Rayo y Becker (2010)- las estrategias de planificación y regulación de la compra podrían no ser suficientes para el consumo habitual que satisface las necesidades y expectativas de los habitantes de la ciudad.

Según la organización Pereira, cómo vamos (2015), en el 2014 se registró una tasa desempleo del 13,7 \% y un índice de pobreza de $18,1 \%$, con una reducción del $6 \%$ con respecto al 2013. La tasa de desempleo fue superior a la media nacional, pero la de pobreza está por debajo, al igual que el coeficiente GINI. Este último índice se ubicó en 0,472 para el 2014. Estos índices económicos indican que a pesar del desempleo, en Pereira hay, en promedio, menos desigualdad que en el resto del país.

En este contexto, las explicaciones del consumo de la teoría del ingreso relativo (Briceño \& Jiménez, 2013) podrían ser pertinentes. Es decir, las decisiones de consumo de un habitante del área metropolitana de Pereira estarían motivadas por la distribución del ingreso en la población, lo cual impulsaría el gasto a pesar de que los ingresos individuales ocasionados por el empleo formal no alcancen para cubrirlo. A partir de allí, los consumidores acudirían al mercado de empleo y al consumo informal, o buscarían acceso al crédito.

Desde la perspectiva del modelo psicoeconómico (Rodríguez, 2006), se predice que esta situación derivaría en un mayor estado de endeudamiento. Además, estos consumidores tendrán una mayor inclinación o actitud irresponsable hacia el endeudamiento, lo cual se relaciona con una percepción pesimista de sus finanzas que y a largo plazo comprometería su bienestar físico y emocional.

Cierto es que el dinero está relacionado con el bienestar, el estrés y la salud mental de las personas (Ahuvia, 2008; Gardner \& Oswald, 2007). Sin embargo, a pesar de las diversas estrategias que se implementen para la administración del dinero, los individuos no toman decisiones fundamentadas, tal como lo plantean los modelos racionales planteados por diversos economistas (para mayor detalle ver Parrado, 2007). Por consiguiente, los resultados señalan la necesidad de adelantar acciones de educación económica y financiera relacionadas con el crédito y las expectativas de consumo, con el fin de disminuir la presencia de conductas impulsivas y mejorar el bienestar subjetivo, tal como lo proponen Herrera, Estrada y Denegri (2011). 
Aunque el ingreso de la mayoría de las personas es medio-bajo y podrían recurrir al crédito como medio de consumo, al no revisar la mayoría de los consumidores encuestados la información objetiva de las etiquetas, podrían comprar habitualmente artículos de baja calidad o más caros de lo que su presupuesto les permite, sin que se percaten de este problema.

Al hilo de estos argumentos, fenómenos como el desempleo, la desigualdad social y el lento crecimiento económico de Pereira son un riesgo para la salud y el bienestar de los habitantes del área metropolitana. En esta dirección, Pérez, Valencia, González y Cardona (2014) señalan dos oportunidades para el mejoramiento del panorama económico de la región. El primero sería la ampliación de la cobertura de servicios e infraestructura, y el segundo el aprovechamiento de las condiciones geográficas, elemento este ligado a objetos y prácticas características del territorio (Barrera, 2012). Ambas propuestas significan cambios en la utilización del contexto de la ciudad como espacio de relaciones, por lo que seguramente tendrían un efecto importante sobre los hábitos de consumo y el bienestar de las personas que lo habitan.

Varios años de investigaciones señalan que el cambio de comportamiento y la creación de hábitos no comienzan por las intenciones o creencias de los individuos (Sniehotta, Presseau \& Araújo-Soares, 2014). Las intervenciones dirigidas a modificar los hábitos de las personas necesariamente deben empezar por las rutinas cotidianas (Juda et al., 2013), acciones que podrían estar ligadas a eventos, objetos, lugares, personas, actividades o periodos determinados (Lally, Wardle \& Gardner, 2011). Son estos los elementos fundamentales para la estructuración de una cultura ciudadana. Para el caso de Pereira, una cultura con hábitos de consumo responsables con el bienestar de sus ciudadanos.

En conclusión, las autoridades locales deberían tener en cuenta el efecto de las políticas de desarrollo económico sobre los hábitos y el bienestar psicológico de las personas que habitan el área metropolitana de Pereira. Para ello se requiere analizar los perfiles del consumidor de acuerdo con sus condiciones sociodemográficas y sus ingresos y medir el bienestar subjetivo para esclarecer la relación enunciada por los autores antes citados, además de comprender la relación entre el comportamiento frente al contexto económico de consumo y el bienestar de los habitantes de la región.

Por último, al corriente de la cadena de acciones y etapas que componen el consumo, es recomendable extender este estudio sobre los hábitos de uso y desecho de los consumidores del área metropolitana de Pereira, puesto que las repeticiones y automatismos en estas fases finales del consumo tienen un efecto directo sobre el medioambiente, el aprovechamiento de los recursos e indirectamente sobre la salud de las personas.

Para futuros estudios se debería tener en cuenta la ampliación de la muestra. Por otra parte, los estudios económicos podrían basarse en indicadores de deuda, consumo y bienestar durante el sondeo poblacional para dar cuenta del panorama de salud de las personas que habitan un área geográfica particular.

Así mismo, los estudios de salud y bienestar se fundamentarían en aspectos de carácter macroeconómico y en las tendencias coyunturales de la economía, las cuales influyen notoriamente en el comportamiento de los consumidores de acuerdo con el ciclo económico que atraviesa la economía en la época del año que se hizo la encuesta (factores estacionales) y el comportamiento de la tasa de cambio o de la tasa de interés.

\section{Referencias}

Ahuvia, A. (2008). If money doesn't make us happy, why do we act as if it does? Journal of Economic Psychology, 29(4), 491507. doi: http://dx.doi.org/10.1016/j.joep.2007.11.005

Barrera, Y. N. (2012). La cultura material cafetera. Revista Gestión y Región, 13, 129-145.

Briceño, J. (2014). La teoría del ingreso permanente: análisis empírico de 19 países latinoamericanos. Denarius, 27, 43-64.

Briceño, J., \& Jiménez, G. (2013). El consumo y la teoría del ingreso relativo. Denarius, 26, 87-114.

Briceño, J., Jiménez, G., \& Muñoz, M. (2015). Teorías que resuelven el enigma del consumo. Denarius, 28, 73-111.

Bruneau-Valenzuela, J. E., Marques de Mello, C., \& David Vieira, F. G. (2009). Consumidores: una reflexión sobre los aspectos de la cultura del consumo. Revista de Administração da UNIMEP, 7(1), 105- 121.

Galvis, A. M. B. (2012). Una mirada a la informalidad en el espacio público de Pereira. Revista Grafías, 16, 11-14.

Gardner, J., \& Oswald, A. J. (2007). Money and mental wellbeing: A longitudinal study of medium-sized lottery wins. Journal of Health Economics, 26(1), 49-60. doi: http://dx.doi. org/10.1016/j.jhealeco.2006.08.004

Gardner, B. (2014). A review and analysis of the use of 'habit' in understanding, predicting and influencing health-related behavior. Health Psychology Review, 9(3), 277-295. doi: 10.1080/17437199.2013.876238 
Gardner, B. (2012). Habit as automaticity, not frequency. European Health Psychologist, 14(2), 32-36.

Henao, O., \& Córdoba, J. F. (2007). Comportamiento del consumidor, una mirada sociológica. Entramado, 3(2), 18-29.

Herrera, M., Estrada G, C., \& Denegri, M. (2011). La alfabetización económica, hábitos de consumo, actitud hacia el endeudamiento y su relación con el bienestar psicológico en funcionarios públicos de la ciudad de Punta Arenas. Magallania, 39, 83-92.

Lally, P., \& Gardner, B. (2011). Promoting habit formation. Health Psychology Review, 7(1), 137-158. doi: 10.1080/17437199.2011.603640

Lally, P., Wardle, J., \& Gardner, B. (2011). Experiences of habit formation: A qualitative study. Psychology, Health \& Medicine, 16(4), 484-489. doi: 10.1080/13548506.2011.555774

Müller, L. H. A. (2009) "Então eu fui à luta!": repensando as representaçóes e práticas econômicas de grupos populares a partir de uma trajetória de ascensão social. Política \& Sociedade, 8(1), 145-171.

Ocampo, M. B. (2014). Las decisiones financieras de la población pobre en Colombia y su importancia en la reducción de pobreza (Tesis de grado inédita). Universidad del Valle, Cali.

Ortega, V., \& Rodríguez-Vargas, J. C. (2004). Escala de hábitos y conductas de consumo: evidencias sobre dimensionalidad. International Journal of Clinical and Health Psychology, 4(1), 121-136.

Ortega, V., Vargas, J. C. R., Denegri, M., \& Gempp, R. (2005). Segmentación psicoeconómica: obtención y validación de perfiles para consumidores adultos jóvenes de nivel socioeconómico medio y alto en Colombia. Revista Internacional de Ciencias Sociales y Humanidades, 15(2), 117-132.

Parrado, F. (2007). Comportamiento de los agentes económicos. Pensando Psicología, 4(5), 25-31.

Pereira cómo vamos. (2015). Informe de calidad de vida 2011 2014. Recuperado de http://goo.gl/0bj8BB
Pérez, G. J., Valencia, F., González, B., \& Cardona, J. C. (2014). Pereira: contexto actualy perspectivas (Documento de trabajo sobre economía regional No. 208). Cartagena: Banco de la Republica de Colombia.

Rayo, L., \& Becker, G. (2007). Habits, Peers, and Happiness: An Evolutionary Perspective. American Economic Review, 97(2), 487-491.

Rodríguez, J. C. (2006). Validación del modelo psicoeconómico del consumidor. Pensamiento \& Gestión, 20, 1-54.

Rubiano, C. X. G. (2010). Pereira imaginada 2009-2014 Fase II ¿La ciudad del deseo o la ciudad del corazón partío? Revista Miradas, 1(9), 88-102.

Schiffman, L. G., \& Kanuk, L. L. (2005). Comportamiento del consumidor. México: Pearson Educación.

Sniehotta, F. F., \& Presseau, J. (2012). The habitual use of the self-report habit index. Annals of Behavioral Medicine, 43(1), 139-140. doi:10.1007/s12160-011-9305-x

Sniehotta, F. F., Presseau, J., \& Araújo-Soares, V. (2014). Time to retire the theory of planned behaviour. Health Psychology Review, 8(1), 1-7. doi: 10.1080/17437199.2013.869710

Solomon, M. R. (2008). Comportamiento del consumidor. Mexico: Prentice Hall.

Velandia, A. \& López-López W. (2008). Investigación cualitativa y psicología del consumidor: alternativas de aplicación. Avances en Psicología Latinoamericana, 26(2), 290-303.

Virla, M. Q. (2010). Confiabilidad y coeficiente Alpha de Cronbach. Telos: Revista de Estudios Interdisciplinarios en Ciencias Sociales, 12(2), 248-252.

Verplanken, B., \& Wood, W. (2006). Interventions to Break and Create Consumer Habits. Journal of Public Policy \& Marketing, 25(1), 90-103.

Wood, W., \& Neal, D. T. (2009). The habitual consumer. Journal of Consumer Psychology, 19(4), 579-592. doi: http:// dx.doi.org/10.1016/j.jcps.2009.08.003 